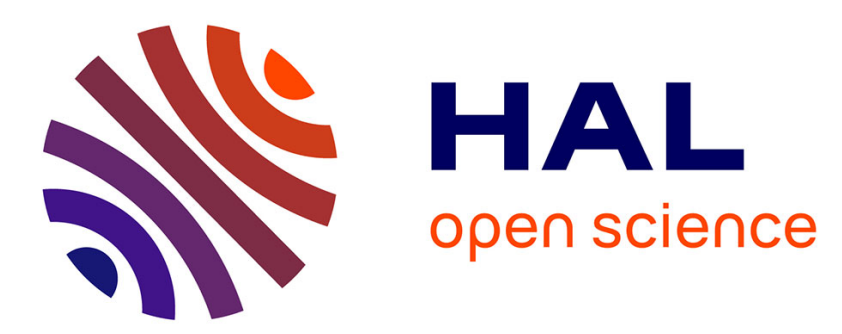

\title{
On the Role of Proprioception in Making Free Throws in Basketball
}

Violaine Sevrez, Christophe Bourdin

\section{To cite this version:}

Violaine Sevrez, Christophe Bourdin. On the Role of Proprioception in Making Free Throws in Basketball. Research Quarterly for Exercise and Sport, 2015, 86 (3), pp.274-280. 10.1080/02701367.2015.1012578 . hal-01429962

\section{HAL Id: hal-01429962 \\ https://hal-amu.archives-ouvertes.fr/hal-01429962}

Submitted on 11 Mar 2019

HAL is a multi-disciplinary open access archive for the deposit and dissemination of scientific research documents, whether they are published or not. The documents may come from teaching and research institutions in France or abroad, or from public or private research centers.
L'archive ouverte pluridisciplinaire HAL, est destinée au dépôt et à la diffusion de documents scientifiques de niveau recherche, publiés ou non, émanant des établissements d'enseignement et de recherche français ou étrangers, des laboratoires publics ou privés.

\section{(c)(1)}

Distributed under a Creative Commons Attribution| 4.0 International License 


\title{
RESEARCH NOTE
}

\section{On the Role of Proprioception in Making Free Throws in Basketball}

\author{
Violaine Sevrez \\ Lyon I University \\ Christophe Bourdin \\ Aix Marseille University
}

\begin{abstract}
Purpose: The aim of this article was to investigate the role that proprioception of the upper limb plays in making basketball free throws. Method: We designed an experiment to directly correlate the performance of basketball players in a free-throw task and an elbowand wrist-joint position sense task. Results: We found a moderately high correlation between the free-throw success rate and wrist-joint position sense and a moderate correlation between the free-throw success rate and elbow-joint position sense. In both cases, the most successful shooters also had the best proprioceptive results. Conclusions: The results indicate that free-throw success is, at least partly, determined by players' ability to sense the position of the distal joints of their throwing upper limb. From a motor-control point of view, this suggests that basketball players may organize the compensatory behavior between the joints of their free-throwing arm on the basis of proprioception. From a practical point of view, it points toward new training techniques to enhance free-throwing efficiency.
\end{abstract}

Keywords: compensatory variability, joint position sense, sensory control, shooting accuracy

Perhaps due to the paradox inherent in the contrast between the apparent ease of shooting free throws in basketball and their troubled success rate, this task has drawn the attention of scientists from various fields during the past decades.

Engineers and physicists first investigated the theoretically optimal basketball trajectories that would distinguish free-throw success from failure through the development of the equations that govern the motion of a projectile. Sports biomechanists set up systematic experiments with players to address temporal, positional, and velocity features adopted by basketball players during a successful free-throw shot. Motor-control specialists have recently considered that movement variability could be beneficial as it gives

Correspondence should be addressed to Violaine Sevrez, CRIS EA 647, Université de Lyon, 27-29, boulevard du 11 novembre 1918, 69622 Villeurbanne Cedex, France. E-mail: violaine.sevrez@univ-lyon1.fr flexibility to being successful when performing the task in spite of potential perturbations or constraints (see Bartlett, Wheat, \& Robins, 2007, for a review on this topic). In this line of thought, Button, MacLeod, Sanders, and Coleman (2003) and Robins and his colleagues (Robins, Davids, Bartlett, \& Wheat, 2008; Robins, Wheat, Irwin, \& Bartlett, 2006) provided support for cooperative behavior between joints of the shooting arm, whereby erroneous deviations of more proximal limb segments can be offset by the more distal joints through the so-called "compensatory variability" (Bootsma \& van Wieringen, 1990). The perceptual processes by which such a compensatory variability is implemented remain to be investigated, but proprioception could be a serious candidate.

Proprioceptive information has been shown to be critical for the control and coordination of goal-directed multijoint movements (Wright, Adamo, \& Brown, 2011) and is particularly required for controlling proximally produced 
joint interaction torques when visual information about the hand is not available (Sainburg, Ghilardi, Poizner, \& Ghez, 1995). Although such situations are commonplace in the field of sports research, focusing on proprioception as it relates to performance is surprisingly still in its infancy. An early study attempted to investigate the relationship between proprioception and physical practice by examining whether knee-joint position sense (JPS) is more accurate in participants who are highly trained in a variety of sports compared with those in an untrained control group (Euzet \& Gahery, 1995). Researchers reported that this was indeed the case, but the data concerned a joint not specifically involved in the sports under examination (i.e., gymnastics, dance, football, archery) and sport level was not considered. Researchers have only very recently addressed the possible link between proprioceptive status and sport achievement. This was done in relation to basketball, through assessing the relationship between successful free-throw percentage and JPS at the knee and shoulder (Kaya, Callaghan, \& Doral, 2012). A moderate correlation $(r=-.60, p=.05)$ was reported between the free-throw percentage and the shoulder JPS at $160^{\circ}$, but the elbow and wrist joints were not investigated.

Our aim was to explore the role of wrist- and elbow-joint proprioception in making free throws in basketball. We therefore designed an experimental protocol to directly correlate the performance of basketball players on a freethrow-shooting task and a joint-positioning task. It was hypothesized that shooting scores would differ depending on players' JPS, with poor shooters exhibiting poorer elbow and wrist proprioception compared with superior shooters.

\section{METHOD}

\section{Participants}

Participants were recruited according to the following three inclusion criteria: (a) varsity basketball team member, (b) right-hand thrower, and (c) no history of neurological conditions or musculoskeletal injuries to the throwing upper limb. Twenty-one male $(n=10)$ and female $(n=11)$ players gave their voluntary informed consent for inclusion in the study, which was approved by the local ethics committee. Their average $( \pm S D)$ age, standing height, and seated height were $20.7 \pm 1.8$ years, $175.3 \pm 11.6 \mathrm{~cm}$, and $133.4 \pm 5.3 \mathrm{~cm}$, respectively. Each participant was tested in a free-throw-shooting task and a JPS task lasting about $30 \mathrm{~min}$ each and respectively designed to evaluate shooting accuracy and proprioception at the wrist and elbow joints.

\section{Evaluation of Shooting Accuracy: Free-Throw- Shooting Task}

In line with our experimental inquiry into the role played by distal upper-limb joints' proprioception in the regulation of free-throw shooting, participants were asked to perform both normal-standing free throws and constrained seated free throws while relying only on movements by the upper limb. Standing trials were performed using the International Basketball Federation regulations (Federation International Basketball Association Central Board, 2004) with official men's and women's basketball sizes for men and women, respectively. In the seated condition, basket height (Bse) was adjusted to shoulder height (Sse) for each participant to maintain the ratio measured in the standing condition between shoulder height (Sst) and basket height (Bst; i.e., Bse/Sse = Bst/Sst; see Figure 1), and shooters were moved backward to maintain a distance of $4.6 \mathrm{~m}$ from the basket. After two or three trial shots to allow for adaption to the experimental situation, participants were asked to perform 50 standing and 50 seated free throws with no time constraints. Standing trials were split in two series, one before the seated trials and one after, to track down any occurrence of fatigue induced by the in-between seated series. The goal of each attempt was to pass the ball through the rim using the overhand push style. Performance was assessed on an all-or-nothing binary basis as it would be in a game situation, with 1 point attributed for a shot that scored and no points attributed for missed shots. The success rate

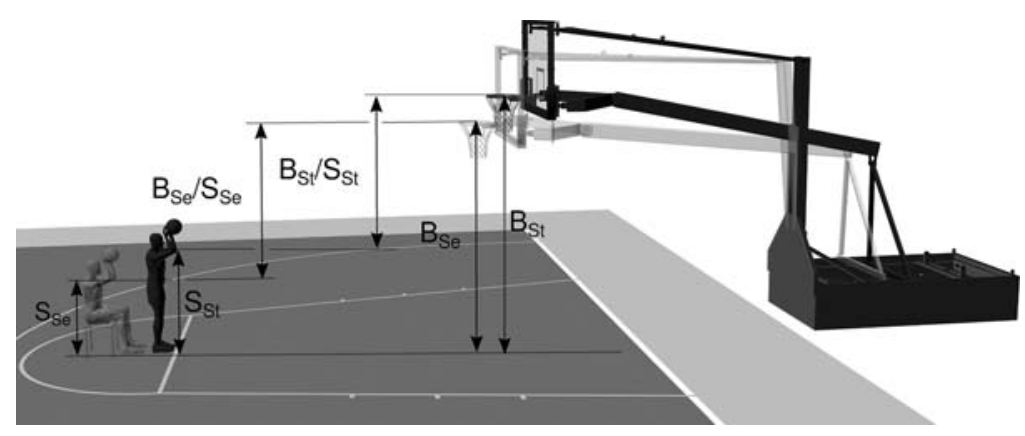

FIGURE 1 Experimental setup for the free-throw-shooting task. Note. Bse = basket height in the seated condition; Bst $=$ basket height in the standing condition; Sse = shoulder height in the seated condition; Sst = shoulder height in the standing condition. 
was then computed for each of the two standing series and for the seated series.

\section{Evaluation of Proprioception at the Wrist and Elbow: Joint Position Sense Task}

Although other methods are available for the assessment of proprioceptive acuity, the joint position sense (JPS) task is the consecrated method for experimental assessment of the accuracy of the system to detect body segment positions and orientations with respect to each other. The JPS task evaluates the ability of participants with their eyes closed or blindfolded to actively or passively reproduce a given joint angle with the same (ipsilateral) or the other (contralateral) limb. Based on the results of previous studies on the validity and reliability of JPS tests (Goble, 2010; Proske \& Gandevia, 2009), we opted here for ipsilateral active matching and ensured that tested amplitudes were of matched magnitude $\left(60^{\circ}\right.$ to $80^{\circ}$ depending on the trials) and that they were established at matched speeds $\left(5^{\circ}\right.$ to $10^{\circ}$ depending on the trials) across all participants. Because JPS is mediated not only by joint and muscle receptors, but also by visual, vestibular, and tactile input, participants were equipped with blindfolds, had their heads fixed, and wore sleeveless T-shirts to remove any extra sources of information. Likewise, reference angles were selected from within the free-throwing task workspace and the extremes of the joints' range of motion were avoided so as to minimize sensory input from cutaneous receptors (Burke, Gandevia, \& Macefield, 1988) and tension on the restraints to movement (Janwantanakul, Magarey, Jones, \& Dansie, 2001). The paradigm involved a custom-made testing apparatus allowing near-frictionless flexion-extension movements about the wrist and elbow joints in the sagittal plane while keeping the shoulder fixed at $90^{\circ}$ flexion (Figure 2). Participants were seated in an adjustable chair, with their right hand grasping a handle and their right forearm and arm being above two aluminum levers. The system was adjusted so that its pivot points were congruent with the wrist- and elbow-joint rotation axes for each participant. While one of the joints was being tested, the other was locked in the neutral position $\left(0^{\circ}\right.$ for the wrist, $90^{\circ}$ for the elbow) with an electro-magnet. After one trial intended to familiarize participants with the task, players' ability to actively reproduce the wrist- and elbow-joint positions was tested five times each in each of three positions (neutral and $30^{\circ}$ of flexion and extension from those positions) in random order. From a given starting position, the experimenter moved the tested joint passively to one of the three reference angles, held it there for $5 \mathrm{~s}$ to enable the participant to become aware of the target angle without tiring, and moved it to another position at a different velocity. Participants were then required to actively move their joint back to the reference angle and to indicate when they felt that they had reached it by pressing a button held in their left hand. Joint rotations were measured using potentiometers mounted beneath the pivot point of the apparatus. Potentiometer resolution was $0.05^{\circ}$; percentage linearity was \pm 0.02 of full range $\left(180^{\circ}\right)$. Each was connected to an analogue-to-digital converter. Signals were registered using LabView (National Instruments Corporation, Austin, TX) and were recorded on a computer. Data were digitized at $100 \mathrm{~Hz}$ and were low-pass filtered (fourth-order Butterworth, zero phase lag, $6 \mathrm{~Hz}$ cutoff frequency). Two types of errors were computed for each trial: (a) the absolute error (AE), which was the mean absolute angular difference between the reference and actual limb position; and (b) the variable error (VE), which was the standard deviation of the five measurements.

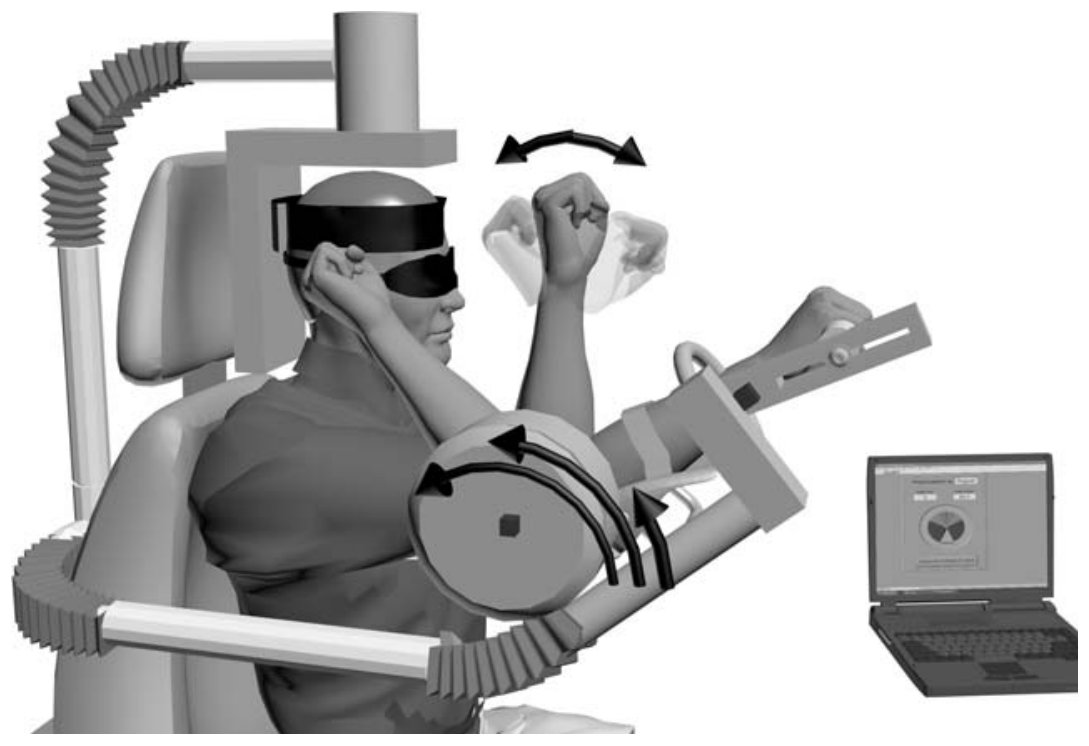

FIGURE 2 Experimental setup for the joint position sense task. 


\section{Data Analysis}

Paired $t$ tests were used to determine if there were differences in the success rate between the two series of standing free throws and between the standing and seated series. Repeated-measures analyses of variance (ANOVAs) were then conducted on both $\mathrm{AE}$ and VE of JPS to determine the main and interaction effects of tested angles (neutral angle and $30^{\circ}$ of flexion and extension from this position) and joints (elbow, wrist). Pearson productmoment correlation coefficients were used as a measure of the degree of fit between the success rate in the standing and seated free-throw conditions and JPS AE and VE measured at the elbow and wrist. As suggested by Zhu (2012), confidence intervals were computed for all variables being tested, correlations were interpreted based the absolute criterions he reported, and inferential statistics were supplemented with measures of effect size $\left(\eta^{2} p\right)$ to quantify the meaningfulness of the differences.

\section{RESULTS}

\section{Free-Throw-Shooting Task}

The standing free-throw success rates ranged widely, from $40 \%$ to $82 \%$, with an average success rate of $62.7 \pm 11.6 \%$ during the 50 trials performed by each player. Success rates in the 25 free throws performed before $(62.9 \pm 12.8 \%)$ and after $(62.8 \pm 12.2 \%)$ the seated series were not different from each other, $t(20)=0.04, p=.96(95 \%$ CI $[-4.33$, $4.52]$ ), thus enabling us to rule out any effect of fatigue. Set $305 \mathrm{~cm}$ high for the standing condition as per the Official Basketball Rules, the basket had to be lowered by $89 \pm 9.5 \mathrm{~cm}$ on average for the seated condition for the ratio between shoulder height and basket height to be maintained. Notwithstanding the fact that standing basketball shots involve multisegment coordination, which includes the trunk and lower body, each individual participant was able to score free throws in this seated condition, with an average success rate of $59.7 \pm 12.5 \%$ during the 50 trials performed in this unusual position. A paired $t$ test was performed to compare the success rate between the standing $(62.7 \pm 11.6 \%)$ and seated $(59.7 \pm 12.5 \%)$ throwing conditions, and this test failed to reject the null hypothesis at the default $\alpha=.05$ significance level, $t(20)=-1.31, p=.20$ (95\% CI $[-7.77,1.77])$, suggesting that the two samples come from distributions with equal means.

\section{Joint Position Sense Task}

The JPS AE and VE for the wrist and elbow joints were $6.2 \pm 2.8^{\circ} / 6.9 \pm 3.6^{\circ}$ and $5.1 \pm 2.6^{\circ} / 5.8 \pm 3.1^{\circ}$ for the neutral angle, $4.8 \pm 2.1^{\circ} / 5.6 \pm 2.4^{\circ}$ and $4.7 \pm 2.5^{\circ} \%$ $4.5 \pm 2.8^{\circ}$ for $30^{\circ}$ of flexion, and $5.4 \pm 2.4^{\circ} / 5.3 \pm 2.8^{\circ}$ and $4.6 \pm 2.0^{\circ} / 5.1 \pm 2.4^{\circ}$ for $30^{\circ}$ of extension, respectively. The ANOVA performed on JPS AE revealed no main effect of angle $(p=.08)$ or joint $(p=.08)$. The ANOVA performed on the JPS VE similarly did not revealed a main effect of angle ( $p=.06)$ but revealed a main effect of joint, $F(1,20)=4.7, p=.04, \eta^{2} p=.189$, with greater variability at the wrist joint than at the elbow joint as revealed by Tukey's post-hoc analysis.

\section{Correlation Between Performance in Free-Throw Shooting and Joint Position Sense Tasks}

The Pearson product-moment correlation coefficients revealed no or low relationships between performance in the standing free-throw-shooting task and JPS at the wrist $(r=-.23, p=.31$ for $\mathrm{AE}$, and $r=-.15, p=.51$ for VE) or elbow joints $(r=-.07, p=.74$ for AE, and $r=-.001$, $p=.99$ for $\mathrm{VE}$ ) but conversely revealed relationships between performance in the seated free-throw-shooting task and JPS. As illustrated in Figure 3, we indeed found a moderately high negative correlation between the seated free-throw success rate and wrist JPS expressed both as AE $(r=-.61, p=.003,95 \%$ CI $[-0.82,-0.24])$ and VE $(r=-.63, p=.002,95 \%$ CI $[-0.83,-0.27])$, and we found a moderate negative correlation between free-throw score and elbow JPS expressed both as AE $(r=-.50$, $p=.02,95 \%$ CI $[-0.77,-0.09])$ and $\operatorname{VE}(r=-.53$, $p=.01,95 \%$ CI $[-0.78,-0.13])$.

\section{DISCUSSION}

Cumulative insight gained on free-throw shooting during the past decades has progressively led to the suggestion that expert performers exploit variability in a functional manner to attain successful free throws under differing task, environmental, or bodily constraints. The perceptual processes by which such a compensatory variability is implemented remain unknown, but proprioception is a good candidate. We therefore set out to determine whether shooting success rate depends on players' JPS accuracy. Because compensation is particularly expected in the distal joints at the end of the movement execution, focusing on the elbow and wrist joints seemed reasonable. The results revealed no correlation between the standing free-throw score and joint position, but they revealed moderate and moderately high correlations between seated free-throw scores and elbow and wrist JPS, respectively.

There were no differences in success rate between the standing and seated free-throwing conditions. However, the 95\% confidence intervals revealed that while the success rate could decrease almost twice as much from standing to seated (up to - 7.77) than from one standing condition to the other (up to -4.33), it could increase by far less from standing to seated (up to 1.77) than from one standing 
condition to the other (up to 4.52). Although not our principal focus, this result may explain why JPS at the elbow and wrist joints was correlated with seated and not standing free-throw scores. Indeed, given that the upper body's contribution to propulsion increases at the end of the propulsion phase and the lower body is the main contributor in the early propulsion phase (Tsarouchas, Kalamaras, Giavroglou, \& Prassas, 1988), removing lower-limb action will decrease overall energy production. Further, considering that the shooting action is a compromise between the allowable margin for error and energy expenditure as reported by Bartlett and his colleagues (2007), the decrease in energy could be detrimental for performance even if freethrow shooting requires submaximal velocity for most populations of players, as this would decrease the margin for error. The two free-throw conditions tested therefore appear to differ in their accuracy demand at the critical release instant. In this way, players relying more on accuracy would perform similarly in the seated condition as in the standing condition in spite of a decreased margin for error resulting from decreased energy production, while players relying more on energy would perform worst in the seated condition. This hypothesis remains to be confirmed through the use of a more sensitive free-throw-shooting rating scale instead of the all-or-nothing binary scale employed here.
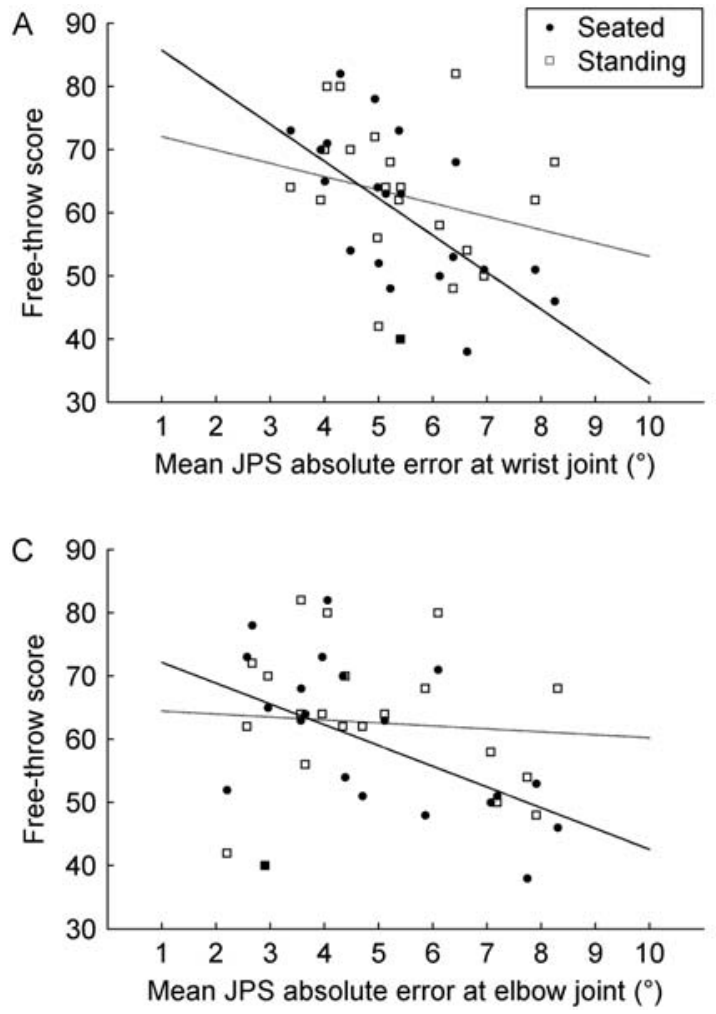

Our principal focus here, though, was to explore the role of dominant upper-limb distal-joint proprioception in seated free-throw performance. The major finding in this line was the relationship between players' seated freethrow score and the JPS at the wrist and elbow joints, with better shooters showing both a more accurate and a less variable position sense than poor shooters. This result extends to players ranging widely in proficiency within a given sport and to the conclusions previously drawn for extreme participants (i.e., participants highly trained in various sports compared with an untrained control group; Euzet \& Gahery, 1995) regarding the relationship between physical practice and JPS. It further gives an early insight into the perceptive mean by which free-throw shooting is controlled. As with any multijoint movement, intersegmental dynamics come to the fore in the free throw, with each body segment affecting the motion of all the other segments in the chain (Hunter, Marshall, \& McNair, 2004). According to Bernstein (1967) and as later confirmed for numerous multijoint movements (Furuya \& Kinoshita, 2007; Sevrez, Rao, Berton, \& Bootsma, 2012), "the secret of coordination lies not only in not wasting superfluous force in extinguishing reactive phenomena but, on the contrary in employing the latter in such a way as to employ active muscle forces only in the capacity of complementary
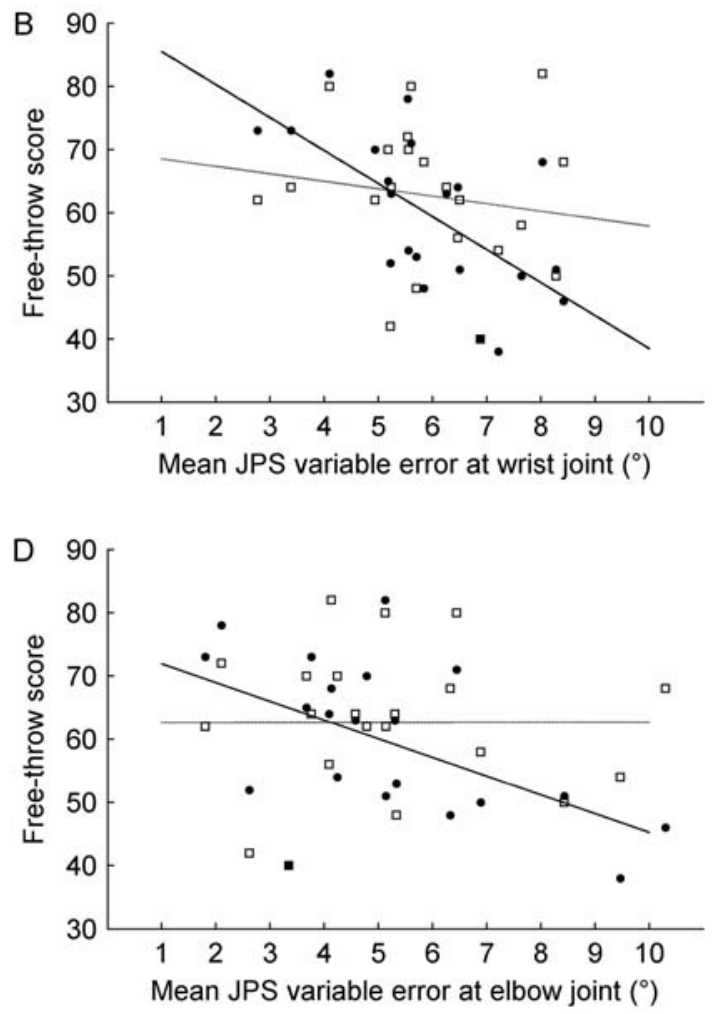

FIGURE 3 Correlation between free-throw score and joint position sense (JPS) at the wrist (upper panels) and elbow (lower panels) expressed as absolute (left panels) and variable (right panels) errors. 
forces" (p. 109). The proximal-to-distal sequencing pattern observed in shooting a basketball (Hayes, 1987), with each segment starting its motion at the instant of greatest speed of the preceding segment and reaching greater speed than that of its predecessor, supports this principle. A reactive phenomenon of this kind has already been shown to play a structuring role in determining the adopted pattern of movement (Putnam, 1993). All of the joints thus contribute to the speed of the distal end of the linked system (hand) by summing their individual speeds through the use of interaction between the joints. In addition to speed demands, the adopted coordination is linked to accuracy demands through compensatory variability (Robins et al., 2008). Thus, while the proximal joints are responsible for the build-up of force, the distal joints help to regulate the velocity and angle of projection of the ball, thereby leading to adequate ball release parameters. As all of the joints except the elbow and wrist are reported to be approaching full extension at the release point, those two joints appear to be instrumental in a free thrower's accuracy, with the hand finally providing the latter compensatory variability just before the release. The fact that the correlation between proprioception and free-throw success rate was moderately high for the wrist joint but only moderate for the elbow joint corroborates the idea that proprioception could serve as a perceptual support for the compensatory variability, whereby erroneous deviations of more proximal limb segments can be offset by more distal joints. Arguments have previously been proposed about the particular role of the wrist in facilitating shooting performance (Robins et al., 2006). The wrist demonstrates a large range of motion for skilled performers and so has a favorable window by which to correct and compensate for previous movements at the more proximal joints, such as the elbow and shoulder.

\section{WHAT DOES THIS ARTICLE ADD?}

This article examined the relationship between performance in free throws and proprioception at the distal joints of the throwing arm to gain insight into the perceptual mechanisms by which basketball players may acquire information that leads to effective performance. Free-throwshooting accuracy is shown to be correlated with wrist and elbow JPS.

From a motor-control point of view, this finding suggests that the compensatory variability process employed in the production and control of the free-throw task may, at least partly, be determined by the quality of afferent proprioceptive input from the wrist joint. From a practical point of view, it represents a first line of evidence to explain why some highly skilled basketball players remain poor shooters after many years of practice, and it points toward new training techniques to enhance throwing efficiency as exercising the proprioceptive system by working on JPS could be beneficial. Further research will be required to clarify whether JPS can be improved by training or whether it is an innate factor that may in itself explain sporting achievement.

\section{ACKNOWLEDGMENTS}

We are grateful to Alain Weisz and Vincent Bousgarbies for sharing their technical expertise in basketball. We would also like to thank Sylvain Raymond, Raphaelle Creniault, Nicolas Flores, Raphael Lagarde, and the players of Aix-Marseille University basketball team for their help in data collection. A very special thanks to Jean-Jacques Temprado for lending us his "coordinator," a multiarticulated coordination apparatus that we have turned into a proprioception-testing apparatus, to Antoine Morice for experimental setup illustrations, and to Michel Poulin for English-language revision.

\section{REFERENCES}

Bartlett, R. M., Wheat, J., \& Robins, M. T. (2007). Is movement variability important for sports biomechanists? Sports Biomechanics, 6 , 224-243.

Bernstein, N. A. (1967). The co-ordination and regulation of movements. Oxford, England: Pergamon.

Bootsma, R. J., \& van Wieringen, P. C. W. (1990). Timing an attacking forehand drive in table tennis. Journal of Experimental Psychology: Human Perception and Performance, 16, 21-29.

Burke, D., Gandevia, S. C., \& Macefield, G. (1988). Responses to passive movement of receptors in joint, skin and muscle of the human hand. Journal of Physiology, 402, 347-361.

Button, C., MacLeod, M., Sanders, R., \& Coleman, S. (2003). Examining movement variability in the basketball free-throw action at different skill levels. Research Quarterly for Exercise and Sport, $74,257-269$

Euzet, J., \& Gahery, Y. (1995). Relationships between position sense and physical practice. Journal of Human Movement Studies, 28, $149-173$.

Federation International Basketball Association Central Board. (2004). Official Basketball Rules, 2004, as approved by Federation International Basketball Association Central Board. Paris, France: Federation International Basketball Association.

Furuya, S., \& Kinoshita, H. (2007). Roles of proximal-to-distal sequential organization of the upper limb segments in striking the keys by expert pianists. Neuroscience Letters, 421, 264-269. doi:10.1016/j.neulet.2007. 05.051

Goble, D. J. (2010). Proprioceptive acuity assessment via joint position matching: From basic science to general practice. Physical Therapy, 90, 1176-1184. doi:10.2522/ptj.20090399

Hayes, D. (1987). Body segment contributions to free throw shooting in basketball. In L. Tsarouchas, J. Terauds, B. A. Gowitzke, \& L. E. Holt (Eds.), Biomechanics in sports V (pp. 205-211). Athens, Greece: Academic Publishers.

Hunter, J. P., Marshall, R. N., \& McNair, P. J. (2004). Segment-interaction analysis of the stance limb in sprint running. Journal of Biomechanics, 37, 1439-1446. doi:10.1016/j.jbiomech.2003.12.018

Janwantanakul, P., Magarey, M. E., Jones, M. A., \& Dansie, B. R. (2001). Variation in shoulder position sense at mid and extreme range of motion. Archives of Physical Medicine and Rehabilitation, 82, 840-844. doi:10. 1053/apmr.2001.21865 
Kaya, D., Callaghan, M. J., \& Doral, M. N. (2012). Shoulder joint position sense is negatively correlated with free-throw percentage in professional basketball players. Isokinetics and Exercise Science, 20, 189-196.

Proske, U., \& Gandevia, S. C. (2009). The kinaesthetic senses. Journal of Physiology, 587, 4139-4146. doi:10.1113/jphysiol.2009.175372

Putnam, C. A. (1993). Sequential motions of body segments in striking and throwing skills: Descriptions and explanations. Journal of Biomechanics, 26(Suppl. 1), 125-135.

Robins, M. T., Davids, K., Bartlett, R. M., \& Wheat, J. S. (2008). Changes in compensatory variability as a function of task expertise and distance during basketball shooting. In Y.-H. Kwon, J. Shim, J. K. Shim, \& I.-S. Shin (Eds.), ISBS - Conference Proceedings Archive, XXV International Symposium on Biomechanics in Sports (pp. 473-476). Seoul, Korea: International Society of Biomechanics in Sports.

Robins, M. T., Wheat, J., Irwin, G., \& Bartlett, R. M. (2006). The effect of shooting distance on movement variability in basketball. Journal of Human Movement Studies, 50, 217-238.
Sainburg, R. L., Ghilardi, M. F., Poizner, H., \& Ghez, C. (1995). Control of limb dynamics in normal subjects and patients without proprioception. Journal of Neurophysiology, 73, 820-835.

Sevrez, V., Rao, G., Berton, E., \& Bootsma, R. J. (2012). On the organizing role of nonmuscular forces during performance of a giant circle in gymnastics. Journal of Applied Biomechanics, 28, 57-62.

Tsarouchas, E., Kalamaras, K., Giavroglou, A., \& Prassas, S. (1988). Biomechanical analysis of free throw shooting in basketball. In E. Kreighbaum \& A. McNeill (Eds.), Proceedings of the 6th International Society of Biomechanics in Sports Symposium (pp. 551-560). Bozeman: Montana State University and the International Society of Biomechanics in Sports.

Wright, M. L., Adamo, D. E., \& Brown, S. H. (2011). Age-related declines in the detection of passive wrist movement. Neuroscience Letters, 500, 108-112. doi:10.1016/j.neulet.2011.06.015

Zhu, W. (2012). Sadly, the earth is still round $(\mathrm{p}<0.05)$. Journal of Sport and Health Science, 1, 9-11. doi:10.1016/j.jshs.2012.02.002 